\title{
A Decade of Editing CNS Spectrums: Back to the Future
}

\author{
By Eric Hollander, MD
}

CNS Spectrums was launched in 1996 as an international journal to address cutting-edge neuroscience developments that address clinical management of psychiatric and neurological conditions. The journal promoted a novel way of looking at disorders by identifying core symptom dimensions linked to underlying brain mechanisms that were expressed across a spectrum of related central nervous system conditions. Hence, the name CNS Spectrums.

I have had the distinct pleasure of being the founding editor, and have served as the editor of this journal off and on for more than 10 years. The last few years have been especially rewarding and a lot of fun to work closely with my very good friends, long-time colleagues Joseph Zohar, MD, the international editor of the journal; Dan J. Stein, MD, PhD; and Stefano Pallanti, MD, PhD. The latter two write terrific columns for the journal ("Pearls in Clinical Neuroscience" and "Brain Stimulation," respectively). It has also been great to see new and successful columns and series launched by Stephen M. Stahl, MD, PhD, and Michael Trimble, MD, FRCP, FRPsych " Trends in Psychopharmacology" and "Brain Regions of Interest," respectively). I would also like to thank Darren L. Brodeur, the publisher of CNS Spectrums, for his determined efforts on behalf of the success of the journal. Finally, I am very pleased that Andrew A. Nierenberg, MD, of Harvard Medical School/Massachusetts General Hospital, has agreed to take on the duties as editor of the journal, beginning in May.

In 1996, we had to struggle to generate enough contributions for a monthly journal. In 2009, our struggle is to efficiently process, triage, accept or reject, and publish the close to nine submitted manuscripts per month. Of note, monthly submissions have nearly tripled in the past 3 years. As a result, time from submission to publication has crept up over the last 3 years from 3.4 months to 5 months. Annual submission rates are shown in Figure 1. The editorial team behind
CNS Spectrums will also soon be taking steps to streamline this process by utilizing ScholarOne, a Web-based approach to standardize, improve, and expedite administrative, editing and reviewing processes, as well as Manuscript Central, a Web-based system for easy administrative, editing, and reviewing capabilities.

The number and percentage of original research submissions continue to rise. In $2008,53 \%$ of submissions were original research, $14 \%$ were review articles, and $33 \%$ were case reports. The journal joined the Neuroscience Peer Review Consortium, an alliance of neuroscience journals that agree to accept manuscript reviews from other members of the Consortium to support efficient and thorough peer review of original research in neuroscience, speed the publication of research reports, and reduce the burden on peer reviewers.

CNS Spectrums is truly an international journal. Last year, $51 \%$ of submissions were from the United States, and $49 \%$ of submissions were international submissions. It is clearly important that our impact factor continues to rise, as it has done steadily, from 2.037 in 2005, to a recent hight of 3.409 (psychiatry)

FIOURE 1.
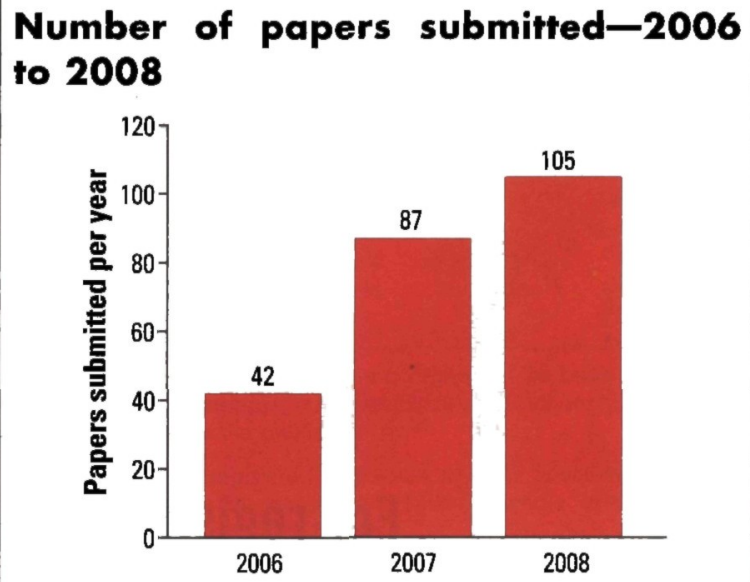

Hollander E. CNS Spectr. Vol 14, No 4. 2009.

Dr. Hollander is the editor of this journal and director of the Institute of Clinical Neuroscience in New York City.

CNS Spectr 14:4 172

April 2009 
in 2007. Likewise, the number of citations continues to rise from 580 in 2005, to 1,179 in 2007 (Figure 2). Of special note, among psychiatry journals, our Impact Factor is now even higher (3.409) (Figure 3).

In 1996, few practitioners or investigators had heard about us. For the CNS Spectrums Web site, the average unique visitors per month increased from 7,098 in 2006, to 28,580 in 2009 (Figure 4). In 2009, the journal is now distributed to 487 domestic and 201 international hospitals and medical school libraries, and mailed to all practicing psychiatrists and neurologists in the US, as well as all CINP members.

The articles in this month's CNS Spectrums focus on adjunctive aripiprazole in treatment-resistant depression; the tolerability profile of desvenlafaxine;

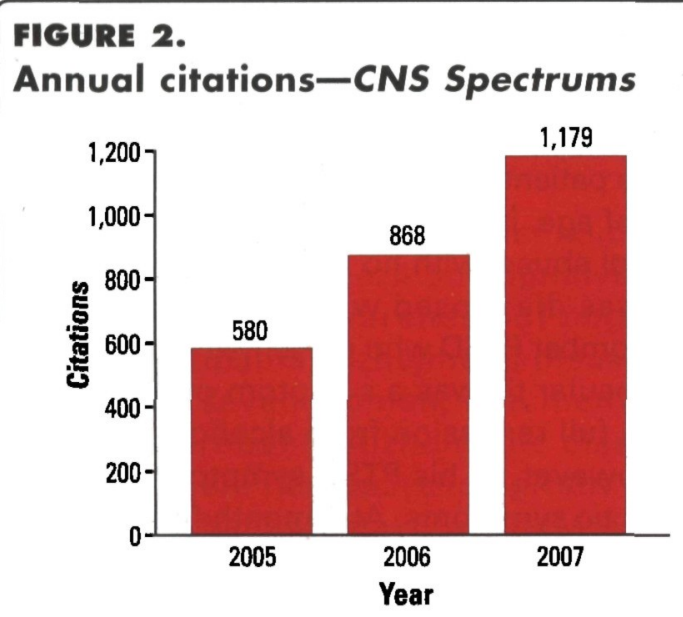

Hollander E. CNS Spectr. Vol 14, No 4. 2009

FIGURE 3.

CNS Spectrums' Journal Citation Reports ${ }^{\oplus}$ Impact Factor

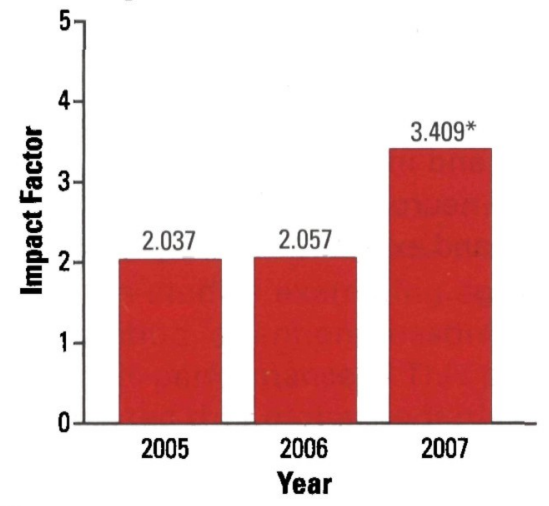

* Psychiatry

Hollander E. CNS Spectr. Vol 14, No 4. 2009 the use of transcranial magnetic theta burst stimulation (TBS) to treat incapacitating tinnitus; and a case of new-onset tics in the context of combat posttraumatic stress disorder (PTSD).

In the current issue, Robert M. Berman, MD, and colleagues evaluated the efficacy and safety of adjunctive aripiprazole versus antidepressant monotherapy in a large sample of patients with major depressive disorder (MDD) and a history of inadequate response to antidepressant monotherapy. Clinically significant improvements in depressive symptoms as assessed by decreases in the Montgomery-Åsberg Depression Rating Scale Total score were greater with adjunctive aripiprazole. Remission rates were greater for adjunctive aripiprazole than for adjunctive placebo $(36.8 \%$ vs $18.9 \%)$. Completion rates with adjunctive aripiprazole and placebo were high ( $83 \%$ vs $87 \%$ ) and discontinuations due to adverse events (AEs) were low $(6.2 \%$ vs $1.7 \%)$. This is the third consecutive clinical trial to demonstrate that aripiprazole augmentation to antidepressants is efficacious for patients with MDD who do not respond adequately

\section{FIGURE 4. \\ Visitor (A) and page-view (B) Web site metrics-Www.cnsspectrums.com}
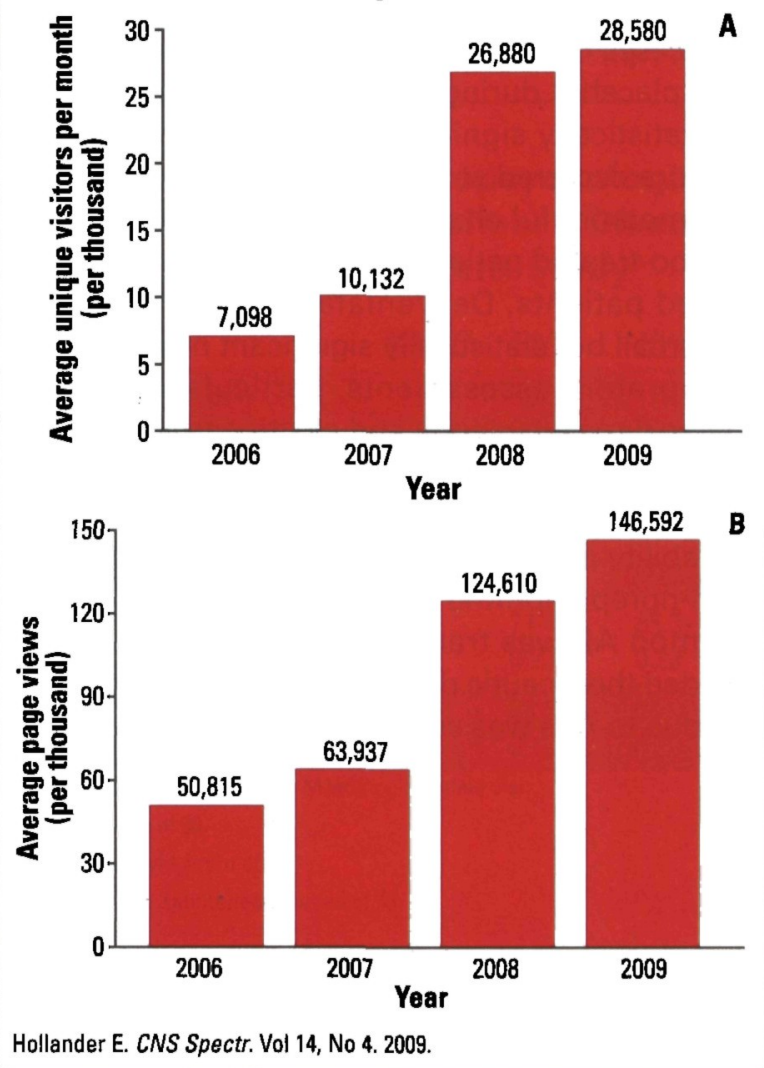
to standard antidepressants. Thus, atypicals are now being demonstrated to have a role as an augmentation strategy in patients who do not have psychosis or bipolar disorder.

Clinicians treating MDD must balance the safety and tolerability profiles versus efficacy profiles of antidepressants in the treatment selection. Anita $\mathrm{H}$. Clayton, MD, and colleagues studied the safety and tolerability of desvenlafaxine in treating depression by analyzing all short-term, randomized, doubleblind, placebo-controlled registration studies for MDD (four flexible-dose and five fixed-dose studies). Adult outpatients with MDD received desvenlafaxine doses ranging from $50-400 \mathrm{mg} / \mathrm{day}$ or placebo for 8 weeks. In the overall population, AEs resulted in discontinuations in $3 \%$ of placebo-treated patients and $12 \%$ of desvenlafaxine-treated patients; in the subset of fixed-dose studies, the rates were $4 \%$ with placebo and increased with desvenlafaxine dose $(50$ $\mathrm{mg} /$ day: $4 \% ; 400 \mathrm{mg} /$ day: $18 \%$ ). The most common treatment-emergent $\mathrm{AE}$ was transient nausea that was generally mild to moderate. The most common sexual dysfunction associated with desvenlafaxine treatment was erectile dysfunction in men $17 \%$ vs $1 \%$ with placebo) and anorgasmia in women $(1 \%$ and $0 \%)$. One desvenlafaxine-treated patient died of a completed suicide; there were four suicide attempts (three desvenlafaxine, one placebo) and eight cases of suicidal ideation (five desvenlafaxine, three placebo) during the on-therapy period. Small but statistically significant changes in mean blood pressure occurred at all desvenlafaxine doses; clinically meaningful changes were observed in $1 \%$ of placebo-treated patients and $2 \%$ of desvenlafaxinetreated patients. Desvenlafaxine was associated with small but statistically significant mean changes in laboratory assessments, particularly lipid and liver enzyme elevations and electrocardiograms. As the authors note in their article, few cases of these changes were clinically relevant. This safety and tolerability profile is generally consistent with serotonin-norepinephrine reuptake inhibitors. The most common AE was transient nausea. At the recommended therapeutic dose of $50 \mathrm{mg} /$ day discontinuation due to AEs was similar to placebo.
Surjo R. Soekadar, MD, and colleagues report a case in which the use of transcranial magnetic TBS treated incapacitating tinnitus accompanied by symptoms of severe depression. Tinnitus is known to be associated with hyperactivity and maladaptive cortical re-organization of the central auditory system. Combined with anxiety and depression it can occasionally constitute a psychiatric emergency. Recently, it has been demonstrated that tinnitus can be temporarily suppressed by noninvasive transcranial magnetic stimulation. TBS is a newly developed technique for rapid and lasting modulation of cortical excitability. In this case, a 54-year-old woman with incapacitating tinnitus had significant improvement in tinnitus and depression after three cycles of 1 -week treatment with continuous TBS to the temporo-parietal auditory association cortex. TBS was an efficient, welltolerated, and practical management of distressing tinnitus accompanied by severe depression.

$\mathrm{CPT}(\mathrm{P})$ Patrick C. Hayes, MD, and colleagues describe a patient with new-onset tics, beginning at 21 years of age, in the context of PTSD and comorbid alcohol abuse, with no known head injury. The patient was diagnosed with alcohol abuse and delayed combat PTSD who presented due to insomnia. The ocular tic was a symptom of PTSD. After achieving full remission from alcohol, the tic persisted. However, as his PTSD symptoms remitted, so did his tic symptoms. At 6-month follow-up, the patient reported he was essentially free from PTSD symptoms, and his tic was significantly reduced.

Thus, this month's issue of CNS Spectrums deals with new uses of atypicals in nonpsychotic illness, tolerability profiles of new antidepressants, new uses of interventional approaches, such as TBS to treat tinnitus, and onset of a tic disorder associated with PTSD.

I have certainly enjoyed my hands-on role as editor of the journal for the past decade and am proud of the journal's continuing improvement in impact factor, the new columns, and the broad influence on innovative and integral issues in neuropsychiatry and clinical neuroscience, and fully expect continuing growth and excellence of the journal. CNS 